\title{
FRACTIONAL EXPONENTIAL DECAY IN THE CAPTURE OF LIGANDS BY RANDOMLY DISTRIBUTED TRAPS IN ONE DIMENSION
}

\author{
- Bernard J. Geurts and Frederik W. Wiegel \\ Center for Theoretical Physics, \\ Twente University, \\ P.O. Box 217, \\ Enschede $7500 \mathrm{AE}$, \\ The Netherlands
}

In many biophysical and biochemical experiments one observes the decay of some ligand population by an appropriate system of traps. We analyse this decay for a one-dimensional system of randomly distributed traps, and show that one can distinguish three different regimes. The decay starts with a fractional exponential of the form $\exp \left[-\left(t / t_{0}\right)^{1 / 2}\right]$, which changes into a fractional exponential of the form $\exp \left[-\left(t / t_{1}\right)^{1 / 3}\right]$ for long times, which in its turn changes into a pure exponential time dependence, i.e. $\exp \left[-t / t_{2}\right]$ for very long times. With these three regimes, we associate three time scales, related to the average trap density and the diffusion constant characterizing the motion of the ligands.

1. Introduction. In many biophysical and biochemical experiments one observes the decay of some ligand population due to capture by an appropriate system of traps. In this paper we consider the decay of an initially homogeneous ligand population due to capture by a one-dimensional system of randomly distributed ideal point traps. As an example, one may think of the diffusion of a number of repressors along a DNA-molecule, followed by their binding to the corresponding operators. The advantage of studying this problem in a strictly one-dimensional geometry is that most questions can be answered analytically.

It will be shown that for such a system, the average ligand decay starts with a square root exponential, i.e. exp[ $\left.-\left(t / t_{0}\right)^{1 / 2}\right]$, which turns into a decay of fractional exponential type for long times, i.e. $\exp \left[-\left(t / t_{1}\right)^{1 / 3}\right]$. This behaviour in turn approaches a decay which is of the pure exponential type, i.e. $\exp \left[-t / t_{2}\right]$, for very long times. Fractional exponential decay types occur in the relaxation of a great variety of complex systems (Ngai and Wright, 1984). Of course, this kinetic behaviour is completely different from the pure exponential decay which is usually assumed in the interpretation of such processes:

In Section 2 we calculate the time dependence of the number of ligands for a special realization of the system, and show that it can be written as the sum of pure exponential decays. In Section 3 the average ligand decay for long systems 
is determined. The decay is characterized by a square root exponential for short times, by another fractional exponential for long times and by a pure exponential for very long times. Some concluding remarks are collected in Section 4.

2. Ligand Decay for a Special Realization of the System. Consider a system of $M$ ideal point traps, randomly distributed on a line segment of length $L$. On this line segment we place $N_{0}(\gg M)$ ligands which are assumed to diffuse along the line. For convenience we assume that the initial ligand distribution is homogeneous. A special realization of the system has traps at $0 \leq x_{1} \leq x_{2} \leq$ $\cdots \leq x_{M} \leq L$. The endpoints $x_{0}=0$, and $x_{M+1}=L$ will be treated as traps as well. Since the system is supposed to be large, boundary effects can be neglected and it is only for convenience that the endpoints are treated as traps.

The ligands are assumed to diffuse along the line; their motion is characterized by a diffusion constant $D$. Essential for this system is that a ligand which initially $(t=0)$ is found located between two traps, will remain there, until it is captured by one of them. Hence, the system decomposes into a large number of small subsystems consisting of a certain number of ligands and two traps at the endpoints, say at $x_{j}$ and $x_{j+1}$.

Let $c_{j}$ denote the number density of ligands in the $j$ th subsystem. One obtains, after introducing the new variable

$$
s=x-\frac{x_{j}+x_{j+1}}{2},
$$

the diffusion equation for the evolution of $c_{j}(s, t)$,

$$
\frac{\partial c_{j}(s, t)}{\partial t}=D \frac{\partial^{2} c_{j}(s, t)}{\partial s_{2}},
$$

with boundary conditions

$$
c_{j}\left(-\frac{1}{2} l_{j}, t\right)=c_{j}\left(\frac{1}{2} l_{j}, t\right)=0
$$

where $l_{j}=x_{j+1}-x_{j}$. Initially, the distribution of ligands is assumed to be homogeneous,

$$
c_{j}(s, 0)=\frac{N_{0}}{L}
$$

for $-\frac{1}{2} l_{j}<s<\frac{1}{2} l_{j}$. The long-time behaviour of the decay of ligands, which will be derived below, is independent of the precise form of the initial distribution. The homogeneous inital situation is assumed only for convenience. It is straightforward to show that the solution of (2)-(4), is given by

$$
c_{j}(s, t)=\frac{4 N_{0}}{\pi L} \sum_{k=1}^{\infty} \frac{(-1)^{k+1}}{(2 k-1)} \cos \left[\frac{(2 k-1) \pi s}{l_{j}}\right] \exp \left[-\left(\frac{2 k-1}{l_{j}}\right)^{2} \pi^{2} D t\right] .
$$


The time dependence of the number of ligands in the jth subsystem $N_{j}\left(t ; l_{j}\right)$ is found from an integration of (5) over the subsystem

$$
N_{j}\left(t ; l_{j}\right)=\frac{8 N_{0}}{\pi^{2} L} \sum_{k=1}^{\infty} \frac{l_{j}}{(2 k-1)^{2}} \exp \left[-\left(\frac{2 k-1}{l_{j}}\right)^{2} \pi^{2} D t\right] .
$$

Upon summing over all subsystems this gives the time dependence of the total number of ligands for the special realization considered

$$
N\left(t ; l_{0}, l_{1}, \ldots, l_{M}\right)=\sum_{j=0}^{M} N_{j}\left(t ; l_{j}\right)
$$

We notice that $N\left(t ; l_{0}, l_{1}, \ldots, l_{M}\right)$ can be written as a sum of pure exponential decays. It should be noted that for any special realization of the system the very long time behaviour is of the pure exponential type, governed by the largest subsystem in that realization.

In any experimental situation one is dealing with a special realization of the system. If $M$ and $L$ are large enough the ligand decay for any special realization can very well be approximated by $\langle N(t)\rangle$, the average of the ligand decay over all realizations of the system, apart from the time dependence for very long times. On the one hand $M / L$ is supposed to be large enough such that in any realization of the system $N\left(t ; l_{0}, l_{1}, \ldots, l_{M}\right)$ is well approximated by $\langle N(t)\rangle$ up to long times. On the other hand, since the system is finite, the very long time behaviour is dictated by the longest subsystem in the particular realization at hand. The average as well as the ultimate time dependence is calculated in the next section and three different time regimes in the decay will be discriminated, as mentioned in the introduction.

3. Average Ligand Decay. In this section we calculate the average ligand decay $\langle N(t)\rangle$ for a large system. The calculation involves basically two steps. First, the probability density function $f(l)$ for the lengths of the subsystems is needed. Second, (6) must be averaged with respect to this weight function $f(l)$. We assume that $N_{0} \gg M \gg 1$. Finally, an estimate for the time dependence for very long times will be given.

Let $P(l)$ denote the probability of finding a subsystem of length $\geq l$, if we distribute randomly $M$ traps on a line segment of length $L$. Consider an interval of length $l$ on this line segment. Since the traps are distributed uniformly this interval can be chosen arbitrarily. If none of the $M$ traps lies on the chosen interval, there is a subsystem with a length at least $l$. The probability that this occurs is given by,

$$
P(l)=\left[1-\frac{l}{L}\right]^{M}
$$


and hence the lengths of the subsystem have the Poisson distribution

$$
f(l)=\frac{M}{L}\left[1-\frac{l}{L}\right]^{M-1} \approx \frac{M}{L} \exp \left[-\frac{M}{L} l\right] .
$$

In terms of $f(l)$ the average ligand decay can be expressed as

$$
\langle N(\tau)\rangle=\frac{8 N_{0}}{\pi^{2}} \sum_{k=1}^{\infty} \frac{1}{(2 k-1)^{2}} \int_{0}^{\infty} y \exp \left[-y-\frac{(2 k-1)^{2} \tau}{y^{2}}\right] \mathrm{d} y
$$

where

$$
y=\frac{M}{L} l ; \tau=\pi^{2}\left(\frac{M}{L}\right)^{2} D t .
$$

The integral in (10a) can not be evaluated analytically. However, one can give accurate approximations for $\langle N(\tau)\rangle$.

For short times the summation involved in (10a) can be approximated as follows. For the function

$$
\varphi(\tau, y)=\sum_{k=1}^{\infty} \frac{1}{(2 k-1)^{2}} \exp \left[-\frac{(2 k-1)^{2} \tau}{y^{2}}\right]
$$

one finds

$$
\begin{aligned}
\frac{\partial \varphi(\tau, y)}{\partial \tau} & =\frac{-1}{y^{2}} \sum_{k=1}^{\infty} \exp \left[-\frac{(2 k-1)^{2} \tau}{y 2}\right] \\
& =-\frac{1}{4}\left(\frac{\pi}{y^{2} \tau}\right)^{1 / 2}\left[1+2 \sum_{k=1}^{\infty}(-1)^{k} \exp \left[-\frac{k^{2} \pi^{2} y}{4 \tau}\right]\right] \\
& \approx-\frac{1}{4}\left(\frac{\pi}{y^{2} \tau}\right)^{1 / 2}
\end{aligned}
$$

if $\tau$ is small enough (Carslaw and Jeager, 1959). Hence, integrating $\partial \varphi / \partial \tau$ one obtains, since $\varphi(0, y)=\pi^{2} / 8$ (Gradshteyn and Rhyzhyk, 1965),

$$
\varphi(\tau, y) \approx \frac{\pi^{2}}{8}-\frac{1}{2}\left[\frac{\pi \tau}{y^{2}}\right]^{1 / 2},
$$

which yields

$$
\begin{aligned}
\langle N(\tau)\rangle & \approx N_{0}\left(1-4 \pi^{-3 / 2} \tau^{1 / 2}\right) \\
& \approx N_{0} \exp \left[-4 \pi^{-3 / 2} \tau^{1 / 2}\right]=N_{0} \exp \left[-4 \frac{M}{L}\left(\frac{D t}{\pi}\right)^{1 / 2}\right] .
\end{aligned}
$$

Hence, we notice that $\langle N(\tau)\rangle$ behaves as a square root exponential for small times. Notice that both the initial value of $\langle N(\tau)\rangle$ as well as the singularity of $\mathrm{d}\langle N(\tau)\rangle / \mathrm{d} \tau$ for $\tau \rightarrow 0$ are correctly represented by (14). 
For moderately long times we can approximate (10a) by using the saddle point method (Copson, 1967). A complete asymptotic analysis of the integral in (10a) can be found in Agmon and Glasser (1986). The saddle point method gives the leading term in the asymptotic expansion. Indeed, changing the integration variable to

$$
x=\tau^{-1 / 3} y
$$

one may write

$$
\langle N(\tau)\rangle=\frac{8 N_{0}}{\pi^{2}} \sum_{k=1}^{\infty} \frac{1}{(2 k-1)^{2}} \tau^{2 / 3} \int_{0}^{\infty} x \exp \left[-\tau^{1 / 3} h_{k}(x)\right] \mathrm{d} x
$$

where

$$
h_{k}(x)=x+\frac{(2 k-1)^{2}}{x^{2}}
$$

The minimum of $h_{k}$ occurs when $x$ equals

$$
x_{0, k}=\left\{2(2 k-1)^{2}\right\}^{1 / 3}
$$

where the second derivative of $h_{k}$ is given by

$$
h_{k}^{\prime \prime}\left(x_{0, k}\right)=6.2^{-4 / 3}(2 k-1)^{-2 / 3} .
$$

Using the saddle point approximation, i.e.

$$
\int_{0}^{\infty} x \exp \left[-\tau^{1 / 3} h_{k}(x)\right] \mathrm{d} x \approx x_{0, k} \exp \left[-\tau^{1 / 3} h_{k}\left(x_{0, k}\right)\right]\left(\frac{2 \pi}{\tau^{1 / 3} h_{k}^{\prime \prime}\left(x_{0, k}\right)}\right)^{1 / 2},
$$

combination of the last six equations yields

$$
\langle N(\tau)\rangle \approx \frac{8}{\sqrt{6}}\left(\frac{2}{\pi}\right)^{3 / 2} N_{0} \tau^{1 / 2} \sum_{k=1}^{\infty} \frac{1}{(2 k-1)} \exp \left[-\tau^{1 / 3}\left\{\frac{27(2 k-1)^{2}}{4}\right\}^{1 / 3}\right]
$$

Approximating the series by the first term one obtains

$$
\begin{aligned}
\langle N(\tau)\rangle & \approx \frac{8}{\sqrt{6}}\left(\frac{2}{\pi}\right)^{3 / 2} N_{0} \tau^{1 / 2} \exp \left[-\tau^{1 / 3}\left(\frac{27}{4}\right)^{1 / 3}\right] \\
& \approx \frac{8}{\sqrt{6}}\left(\frac{8}{\pi}\right)^{1 / 2} N_{0} \frac{M}{L}(D t)^{1 / 2} \exp \left[-\left(\frac{27}{4}\right)^{1 / 3}\left(\frac{\pi M}{L}\right)^{2 / 3}(D t)^{1 / 3}\right],
\end{aligned}
$$

which is valid for long times. As the factor $\tau^{1 / 2}$ becomes irrelevant if $\tau$ becomes large enough the last formula shows that the ligand decay $\langle N(\tau)\rangle$ is of the fractional exponential form. This has been remarked by several authors before (Donsker and Varadhan, 1975; Weiss, 1986), and is the only asymptotic time dependence of the number of ligands for an infinite system. A regime in which 
the decay is proportional to $\exp \left(-\left(t / t_{1}\right)^{1 / 3}\right)$ has been recently observed for one-dimensional systems in measurements of electric-field-dependent, chargecarrier trapping (Seiferheld et al., 1983) and in numerical simulations (Klafter et al., 1984).

In Fig. 1 a numerical evaluation of (10a), together with the approximations (14) and (22) is shown. Notice the accuracy of the small-time approximation for $\tau<5$. The long-time approximation is quite good for $\tau$ of the order 20 and larger. Notice that then about $4 \%$ of the initial ligand population is still present.

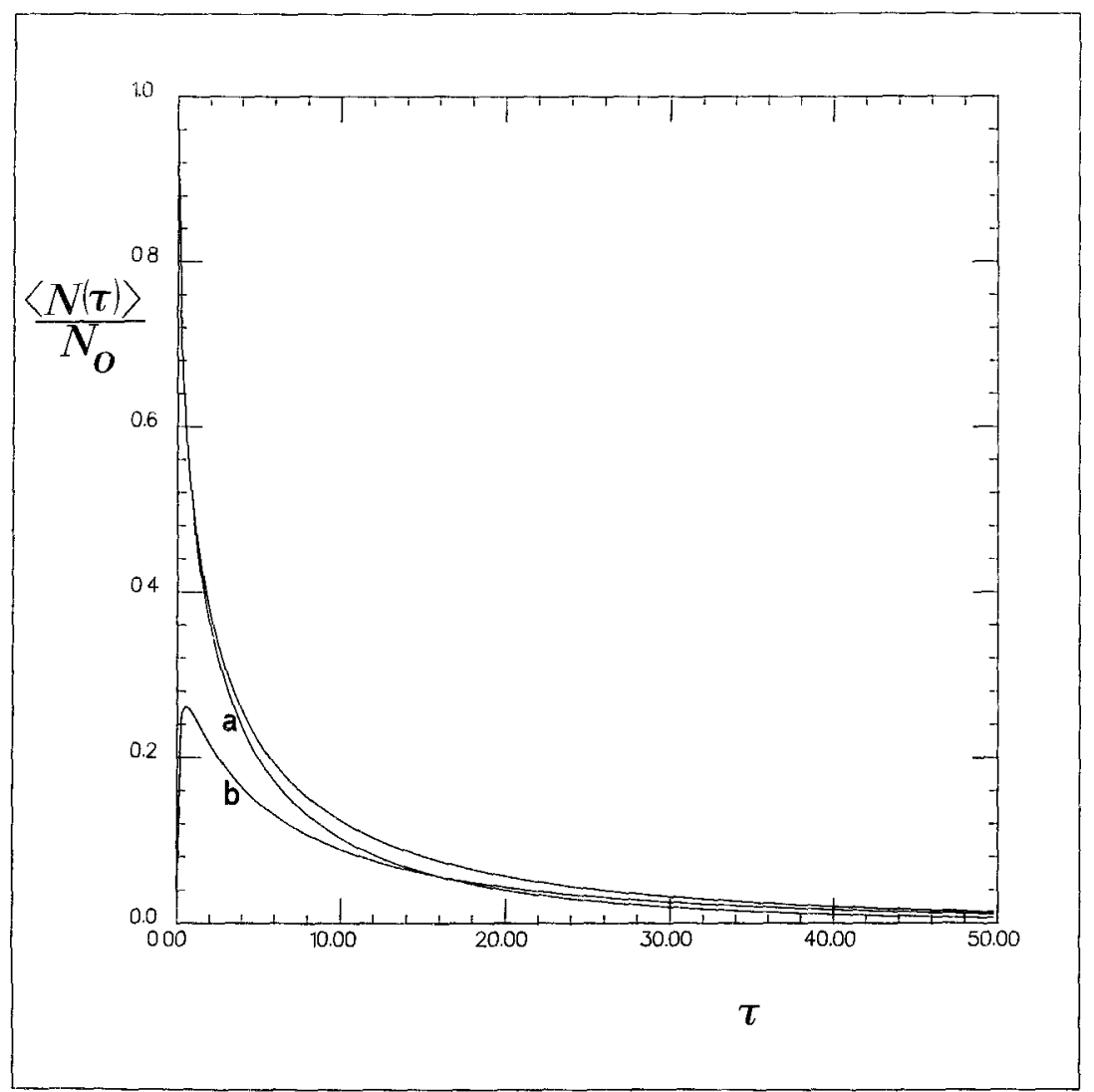

Figure 1. Plot of the ratio between the average ligand population and the initial population $\langle N(\tau)\rangle / N_{0}$ as a function of the dimensionless "time" $\tau=\pi^{2}(M / L)^{2} D t$, where $D$ is the diffusion constant. The line marked with a represents the short-time approximation (14) and the line marked with $\mathbf{b}$, the long-time approximation (22).

As remarked at the end of Section 2, the time dependence of the number of ligands for very long times is dominated by the decay in the largest subsystem. This is of the pure exponential type and varies from realization to realization. It is a consequence of the fact that the system considered is large but finite. We 
roughly estimate the expectation value of the length of the largest subsystem and obtain an estimate of the time dependence for very long times and of the corresponding time scale.

According to (9) one can write the number of intervals with a length larger than $l_{0}$ as

$$
n=M \int_{l_{0}}^{\infty} \frac{M}{L} \exp \left[-\frac{M l}{L}\right] \mathrm{d} l
$$

Hence, if

$$
l_{0}=\frac{L}{M} \ln (M)
$$

one has $n=1$. Thus, on the average there is one subsystem of length $\geq l_{0}$. Denoting by $l_{\max }$ the expectation value of the length of the largest subsystem, one finds, after renormalizing the distribution,

$$
l_{\max }=\frac{L}{M}(\ln (M)+1) \text {. }
$$

Hence, in view of (6) and (7), on the average the very long time decay of the ligands in the system can be approximated by a pure exponential decay with rate constant,

$$
t_{2}=\frac{l_{\max }^{2}}{\pi^{2} D}
$$

Adopting the point of view that this behaviour will set in roughly as $t>t_{2}$ or $\tau>(\ln (M)+1)^{2}$, we can state in addition that the fractional exponential time dependence $(22)$ is, on the average, valid up to $\tau \approx(\ln (M)+1)^{2}$.

4. Concluding Remarks. In many relaxation processes in complex systems, one observes a fractional exponential time behaviour (Ngai and Wright, 1984). We have shown that the decay of an initially homogeneous ligand population due to their capture by a one-dimensional system of randomly distributed ideal traps has a fractional exponential time dependence for moderately long times, i.e. in order of magnitude $20 /\left(\pi^{2}(M / L)^{2} D\right) \leq t \leq(\ln (M)+1)^{2} /\left(\pi^{2}(M / L)^{2} D\right)$, for large systems. The two- and three-dimensional cases will be treated elsewhere (Wiegel and van Opheusden, 1987).

Qualitatively speaking, the ligand decay starts as a square root exponential for times $t<5 /\left(\pi^{2}(M / L)^{2} D\right)$. For $t$ larger than $T_{1} \approx 20 /\left(\pi^{2}(M / L)^{2} D\right)$ but smaller than $T_{2} \approx(\ln (M)+1)^{2} /\left(\pi^{2}(M / L)^{2} D\right)$ the time dependence aproaches a behaviôur proportional to $t^{1 / 2} \exp \left[-\left(t / t_{1}\right)^{1 / 3}\right]$, with $t_{1}=4 /\left(27 \pi^{2}(M / L)^{2} D\right)$, which turns into a pure exponential time dependence for $t \geq T_{2}$ with rate constant given by (26). 
By performing decay experiments of the type mentioned in the introduction and measuring over long enough times, at least the transition from (14) to (22) should be detectable if the trap density $M / L$ is large enough. At a typical value for $D \approx 10^{-10} \mathrm{~m}^{2} / \mathrm{sec}$ and $M / L \approx 10^{4} / \mathrm{m}$ one has $T_{1} \approx 2.10^{2} \mathrm{sec}$. After a few minutes, the fractional exponential decay type predicted should set in. The fraction of ligands not captured yet, is then about $4 \%$. Hence it should be possible to find experimental systems in which the transition from a square root exponential decay to a fractional exponential of type (22) can be detected. The transition to the ultimate pure exponential decay type is much more difficult to observe because the number of ligands will have decayed so far that detection is unlikely. Apart from that, the time scale at which this behaviour sets in will be quite large.

One of the authors (F.W.W.) has profited from discussions with Dr Alan S. Perelson, which were made possible by NATO Collaborative Research Grant $86 / 719$.

\section{LITERATURE}

Agmon, N. and M. L. Glasser. 1986. Phys. Rev. A 34, 656.

Carslaw, H. S. and J. C. Jeager. 1959. Conduction of Heat in Solids, equations 12, 13, p. 97. Oxford: Clarendon Press.

Copson, E. T. 1967. Asymptotic Expansions, pp.91-98. Cambridge: Cambridge University Press.

Donsker, M. D. and S. R. S. Varadhan. 1975. Communs pure appl. Math. 28, 525.

Gradshteyn, I. S. and I. M. Rhyzhik. 1965. Table of Integrals, Series and Products, equation 0.234-2. New York: Academic Press.

Klafter, J., B. Zumofen and A. Blumen. 1984. J. Physique Lett. 45, L-49.

Ngai, K. L. and G. B. Wright (Eds). 1984. Relaxations in Complex Systems. Arlington: Office of Naval Research.

Seiferheld, U., H. Baessler and B. Movaghar. 1983. Phys. Rev. Lett. 51, 813.

Weiss, G. H. 1986. J. Stat. Phys. 42, 3.

Wiegel, F. W. and J. H. J. van Opheusden. 1987. University of Twente, preprint.

RECEIVED 8-5-86

REVISED 2-25-87 